\title{
Análisis dosimétrico entre puntos «A» de Mánchester y volumen blanco clínico de alto riesgo en braquiterapia 3D guiada por imagen para el cáncer cervicouterino
}

\author{
Silvia C. Chacón-Anaya*, Mauricio Durán-Cruz, Arturo Reding-Bernal y José A. Barragán-Pérez \\ Servicio de Radiooncología, Hospital General de México, Secretaría de Salud, Ciudad de México, México
}

\begin{abstract}
Resumen
Introducción: La braquiterapia ginecológica ha evolucionado desde el concepto de prescripción puntual (Mánchester) a volúmenes de tratamiento. Objetivo: Analizar el resultado dosimétrico entre la prescripción a puntos $A$ y volumen blanco clínico de alto riesgo (CTV-HR). Métodos: Se realizaron ambas planeaciones en 28 pacientes con cáncer cervicouterino estadio IB2-IVA (112 aplicaciones), la dosis utilizada fue cuatro fracciones de 5.62 Gy. Resultados: La dosis media al 90\% del CTV-HR y desviación estándar: $92.1 \pm 2.7$ Gy fue más baja $(p<0.008)$ que a puntos $A(103.4 \pm 21.6$ Gy). La cobertura al CTV-HR: $97.8 \pm 7.3 \%$ fue mayor ( $p<0.000000000043)$ que con puntos $A(86.6 \pm 14.7 \%)$. La D2 cc de vejiga fue: $83.8 \pm$ 22.4 Gy vs. punto vejiga ICRU $38(66.1 \pm 7.6 \mathrm{~Gy})(p<0.0057)$, demostrando la subestimación de la dosis en la planeación puntual. La D2 cc de recto fue: $73.6 \pm 12.7$ Gy vs. punto recto ICRU 38 (75.1 \pm 11.9 Gy) $(p<0.4857)$, no difiriendo significativamente. Conclusiones: La braquiterapia 3D proporciona una mayor cobertura de dosis al CTV-HR comparándolo con el sistema tradicional, existiendo una subestimación de la dosis recibida en vejiga en el sistema puntual.
\end{abstract}

Palabras clave: Cervicouterino. Braquiterapia intracavitaria. Mánchester. Volumétrica.

Dosimetric analysis between Mánchester points "A" and high-risk clinical target volume in the three-dimensional image-guided branchytherapy for uterine cervical cancer

\section{Abstract}

Background: Gynecological brachytherapy has evolved from the concept of punctual prescription (Manchester) to treatment volumes. Objective: To analyze the dosimetric result between the prescription at point $A$ and high-risk clinical target volume (HR-CTV). Methods: Both plans were performed in 28 patients with stage IB2-IVA cervical cancer (112 applications), the dose used was 4 fractions of 5.62 Gy. Results: The mean D90 (dose to $90 \%$ volume) and its standard deviation for HR-CTV: $92.1 \pm 2.7$ Gy, was lower $(p<0.008)$ than at points A (103.4 \pm 21.6 Gy). HR-CTV coverage: $97.8 \pm 7.3 \%$ was higher $(p<$ $0.000000000043)$ than with points $A(86.6 \pm 14.7 \%)$. The bladder D2 cC was: $83.8 \pm 22.4$ Gy vs. ICRU 38 bladder point (66.1 $\pm 7.6 \mathrm{~Gy})(p<0.0057)$, demonstrating the underestimation of the dose in the punctual planning. The $D 2 \mathrm{cc}$ of the rectum was: $73.6 \pm 12.7$ Gy vs. ICRU 38 straight point $(75.1 \pm 11.9$ Gy) $(p<0.4857)$, not differing significantly. Conclusions: $3 D$ brachytherapy provides greater dose coverage for HR-CTV compared to the traditional system, with an underestimation of the dose received in the bladder in the punctual system.

Key words: Cervical cancer. Brachytherapy. Intracavitary. Manchester. Volumetric.

Correspondencia:

*Silvia C. Chacón-Anaya

E-mail: silvitaconstanza@gmail.com
Disponible en internet: 27-11-2020 Gac Mex Oncol. 2021;20(1):3-10 www.gamo-smeo.com 2565-005X/C 2020 Sociedad Mexicana de Oncología. Publicado por Permanyer. Este es un artículo open access bajo la licencia CC BY-NC-ND (http://creativecommons.org/licenses/by-nc-nd/4.0/). 


\section{Introducción}

El cáncer del cuello uterino ocupa la cuarta posición mundial en incidencia y mortalidad de cáncer en mujeres ${ }^{1}$. La radioterapia de haz externo y la braquiterapia han sido pilar del tratamiento radical durante más de medio siglo en estadio localmente avanzado².

La braquiterapia intracavitaria consiste en colocar una sonda intrauterina y dos colpostatos vaginales, con posterior carga de fuentes radiactivas ${ }^{3,4}$. El sistema de Mánchester es el más utilizado en el mundo, inició con el uso de imágenes en $2 \mathrm{D}$ (dos dimensiones) ${ }^{5,6}$, mediante radiografías ortogonales, en las cuales se prescribía la dosis a los puntos A de Mánchester ${ }^{7,8}$. Actualmente con el avance de la tecnología, la braquiterapia guiada por imágenes (IGBT) en 3D, con el uso de la tomografía computarizada (TC) y resonancia magnética (RM) han permitido el contorneo del tumor en el cáncer cervicouterino y los órganos sanos: vejiga, recto y sigmoides, con una técnica más precisa, ofreciendo mejoras en los parámetros de dosis-volumen y en el resultado clínico ${ }^{9,10}$.

En este estudio se compararan y determinarán las diferencias existentes en el porcentaje de cobertura de dosis tumoral y las dosis de radiación recibidas por el tumor y los órganos de riesgo recto y vejiga en ambas planeaciones: prescripción de la dosis a los puntos $A$ de Mánchester vs. prescripción de volumen blanco clínico de alto riesgo (CTV-HR, high-risk clinical target volume) en pacientes tratadas con braquiterapia ginecológica de alta tasa de dosis.

\section{Material y métodos}

En 28 pacientes con cáncer cervicouterino estadio IB2-IVA (112 aplicaciones de braquiterapia ginecológica), tratadas en la unidad de radioterapia del Servicio de Oncología del Hospital General de México Dr. Eduardo Liceaga durante el periodo comprendido del 1 de noviembre del año 2017 al 1 de septiembre del año 2018, con braquiterapia de alta tasa de dosis (BQTHDR, high dose rate brachytherapy) mediante arreglo simultáneo (sonda intrauterina con par de colpostatos) utilizando TC para la simulación mediante una planeación en $2 \mathrm{D}^{8}$; de forma alterna, a cada paciente ya tratada se le elaboró otra planeación volumétrica, utilizando las recomendaciones de las Guías GYN GEC-ESTRO;; la dosis prescrita fueron cuatro fracciones de $5.62 \mathrm{~Gy}$, dos veces por semana, para una dosis equivalente a 2 Gy/día (EQD2) con alfa/beta de $10=29.3$ Gy, para el cálculo de la EQD2 en los órganos de riesgo, se utilizó un alfa/beta de 3 . El equipo de braquiterapia utilizado fue ECLIPSE ${ }^{\mathrm{TM}}$ : BrachyVision 3D-gammaMedPlus iX HDR/PR (Varian), utilizando el radioisótopo Iridio-192. En la planeación con prescripción a puntos $A$ de Mánchester se determinaron los puntos de referencia «A", basados en el sistema desarrollado por Tod y Meredith en $1938^{5}$ y los puntos de referencia del recto y vejiga según el ICRU 38 (Informe n. 38 de la Comisión Internacional de Unidades de Radiación y Medición) ${ }^{11}$. En la planeación con prescripción volumétrica, se contorneó el CTV-HR y los órganos de riesgo según las guías GYN GEC-ESTRO. Estas guías determinan que la delineación del GTV (volumen de enfermedad macroscópica visible por técnicas de imagen) y el CTV se debe realizar para cada aplicación de braquiterapia, tomando en cuenta las imágenes de RM en T2 tanto al diagnóstico como en cada aplicación de la braquiterapia. Define el GTV diagnóstico como la extensión macroscópica del tumor al diagnóstico detectada por examen clínico y por T2 de RM. El GTV braquiterapia lo definen como la extensión macroscópica del tumor al momento de la aplicación de braquiterapia detectado por examen clínico y por T2 RM. El CTV-HR (alto riesgo) incluye el GTV braquiterapia con la totalidad del cérvix y la extensión extracervical del tumor sin adicionar márgenes de seguridad $^{9}$. También mencionan que tanto la RM como la TC proveen similar calidad para la discriminación de los órganos de riesgo como vejiga, recto, sigmoides, intestino delgado y vagina, pero con relación a la discriminación del GTV y tejido uterino normal adyacente es superior la $\mathrm{RM}^{10}$.

Eskander, et al. realizaron el contorneo del CTV-HR y los órganos de riesgo con TC y RM con las recomendaciones antes mencionadas, demostrando que si bien la TC sobreestima el CTV-HR en su eje coronal, los parámetros de dosis no mostraron diferencias significativas entre RM y TC, de igual forma en los órganos de riesgo no se evidenciaron diferencias significativas, concluyendo que es factible la aplicación de braquiterapia 3D guiada por imagen utilizando $\mathrm{TC}^{12}$.

En nuestro estudio se obtuvieron imágenes para planeación del tratamiento mediante equipo de TC con cortes de $3 \mathrm{~mm}$ a lo largo de la pelvis. El contorneo del CTV-HR se realizó incluyendo la totalidad del cérvix y su extensión extracervical visibles tanto en la imagen de TC de cada aplicación de braquiterapia como en los hallazgos encontrados en la exploración física. Para los órganos de riesgo se realizó el contorneo de recto, sigmoides, vejiga e intestino delgado. La vejiga fue contorneada desde su base hasta el domo; el recto fue contorneado desde las tuberosidades isquiáticas hasta la flexura del sigmoides; el sigmoides fue contorneado 
Tabla 1. Planeación con prescripción a volumen blanco clínico de alto riesgo (CTV-HR, clinical target volume-high risk)

\begin{tabular}{|l|c|c|c|c|c|c|}
\hline \multicolumn{7}{|c|}{ Dosis total promedio de variables: EBRT + BOT-HDR (EQD2) } \\
\hline N. pacientes & $\begin{array}{c}\text { Volumen } \\
\text { Tumoral (cc) }\end{array}$ & $\begin{array}{c}\text { Dosis recibida por el } \\
\text { tumor D90 } \\
\text { (CTV-HR (Gy) }\end{array}$ & $\begin{array}{c}\text { Porcentaje de } \\
\text { cobertura de dosis al } \\
\text { tumor (CTV-HR) (\%) }\end{array}$ & $\begin{array}{c}\text { Dosis a puntos A } \\
\text { de Mánchester } \\
\text { (Gy) }\end{array}$ & $\begin{array}{c}\text { Dosis a } 2 \text { cc } \\
\text { de vejiga } \\
\text { (Gy) }\end{array}$ & $\begin{array}{c}\text { Dosis a } 2 \text { cc } \\
\text { de recto } \\
\text { (Gy) }\end{array}$ \\
\hline 28 & $43.0 \pm 12.9$ & $92.1 \pm 2.7$ & $97.8 \pm 7.3$ & $103.4 \pm 21.6$ & $83.8 \pm 22.4$ & $73.6 \pm 12.7$ \\
\hline
\end{tabular}

EBRT: external beam radiation therapy (radioterapia de haz externo); BOT-HDR: high dose rate brachytherapy (braquiterapia de alta tasa de dosis); EQD2: dosis equivalente a 2 Gy por fracción; D90: dosis recibida por el 90\% del volumen tumoral; cc: centímetros cúbicos; Gy: Gray.

desde la flexura del sigmoides hasta $2 \mathrm{~cm}$ arriba del aplicador; y el intestino delgado fue contorneado desde la aparición de este en el corte de tomografía más inferior hasta $2 \mathrm{~cm}$ por arriba del aplicador.

Se realizó un estudio observacional, descriptivo, retrospectivo y transversal, en donde se registraron los datos arrojados en los histogramas de dosis y volumen. La dosis total integral (teleterapia + braquiterapia) de cada variable por paciente se obtuvo sumando la dosis de radioterapia externa con la dosis EQD2 de braquiterapia.

El tamaño de la muestra se determinó mediante el método de diferencia de dos medias para muestras pareadas con una hipótesis de dos colas, con un tamaño de muestra del efecto $d$ de Cohen de 0.2 con una potencia del $80 \%$ y una fiabilidad del $95 \%$. La estimación de este tamaño de muestra se determinó mediante el software estadístico G power versión 3.1.9.2.

Se realizó un análisis de los datos obtenidos usando el programa SPSS y Stata 10, comparando las variables en la planeación volumétrica vs. puntual: dosis recibida por el $90 \%$ del volumen tumoral, porcentaje de cobertura tumoral (CTV-HR), punto de vejiga vs. D2 cc (dosis en 2 centímetros cúbicos) de vejiga, punto de recto vs. D2 cc de recto.

\section{Resultados}

En un periodo de nueve meses se recabaron los expedientes clínicos y archivos digitales de 28 pacientes (112 aplicaciones de braquiterapia) con cáncer cervicouterino IB2-IVA, tratados con BQT-HDR, con el sistema tradicional de prescripción puntual (puntos A Mánchester y puntos órganos de riesgo definidos por el ICRU 38) quienes fueron simuladas con TC. La media de edad fue de 46.39 años con desviación estándar de \pm 10.45 . Según el reporte histopatológico, el carcinoma epidermoide fue diagnosticado en 22 pacientes (78\%), el adenocarcinoma en 5 pacientes (18\%) y el carcinoma con celulas en anillo de sello en 1 paciente (4\%). La estadificación FIGO
(Federación Internacional de Ginecología y Obstetricia) fue I en 2 pacientes (7\%), II en 14 pacientes (50\%), III en 5 pacientes (18\%) y IV en 7 pacientes (25\%). La dosis de radioterapia externa fue 50 Gy en 23 pacientes (82\%) y 45 Gy en 5 pacientes (18\%). Las cinco pacientes que recibieron 45 Gy tenían afectación de ganglios paraaórticos. La quimioterapia concomitante a base de cisplatino $\left(40 \mathrm{mg} / \mathrm{m}^{2}\right)$ con radioterapia externa se administro a 26 pacientes $(93 \%)$, no recibió quimioterapia 1 paciente (4\%) y se desconoce si recibió quimioterapia en 1 paciente (4\%). La media del volumen tumoral al momento de la braquiterapia fue $43.03 \mathrm{cc}$ con desviación estándar de \pm $12.95 \mathrm{cc}$; volumen tumoral menor a $20 \mathrm{cc}$ en 1 paciente (4\%), de $20-40 \mathrm{cc}$ en 10 pacientes (36\%) y mayor a 40 cc en 17 pacientes (61\%).

En las 112 planeaciones de BQT-HDR guiada por TC, la prescripción de dosis fue 5.62 Gy por fracción, para un total de 22.48 Gy en cuatro fracciones. La dosis al 90\% del volumen blanco clínico de alto riesgo (CTV-HR D90) fue de 74.26-79.26 Gy, mientras que la dosis a 2 cc de vejiga y recto se limitó a 90 y 75 Gy respectivamente. La dosis a puntos $A$ de Mánchester fue de 74.26-79.26 Gy, y la dosis a punto ICRU 38 de vejiga y recto se limitó a 80 y 75 Gy respectivamente.

En la planeación volumétrica la dosis media al CTVHR D90 fue 92.1 Gy con desviación estándar de \pm 2.7 Gy, significativamente menor $(p<0.00816)$ que la dosis media al punto A de Mánchester 103.4 \pm 21.6 Gy (Tabla 1). En la planeación volumétrica el porcentaje de cobertura de dosis al tumor (CTV-HR) fue $97.8 \pm$ $7.3 \%$, significativamente mayor $(p<0.000000000043)$ que la cobertura de dosis al tumor (CTV-HR) con la planeación a puntos A de Mánchester: $86.6 \pm 14.7 \%$ (Tablas 1 y 2).

Los rangos de dosis de los órganos de riesgo estaban dentro los límites aceptables. La dosis media a 2 cc de recto fue $73.6 \pm 12.7$ Gy. Fue menor y no significativa $(p<0.48572)$ que la dosis media a punto recto (ICRU 38) $75.09 \pm 11.9$ Gy. La dosis media a 2 
Tabla 2. Planeación con prescripción a puntos A de Mánchester

\begin{tabular}{|c|c|c|c|c|}
\hline \multicolumn{5}{|c|}{ Dosis total promedio de variables: EBRT + BOT-HDR (EQD2) } \\
\hline N. ${ }^{0}$ pacientes & $\begin{array}{l}\text { Dosis recibida por el tumor D90 } \\
\text { (CTV-HR) (Gy) }\end{array}$ & $\begin{array}{c}\text { Porcentaje de cobertura de dosis } \\
\text { al tumor (CTV-HR) (\%) }\end{array}$ & $\begin{array}{l}\text { Punto vejiga } \\
\text { ICRU } 38 \text { (Gy) }\end{array}$ & $\begin{array}{l}\text { Punto recto } \\
\text { ICRU } 38 \text { (Gy) }\end{array}$ \\
\hline 28 & $80.2 \pm 8.2$ & $86.6 \pm 14.7$ & $66.1 \pm 7.6$ & $75.9 \pm 11.9$ \\
\hline
\end{tabular}

cc de vejiga fue $83.8 \pm 22.4$ Gy fue significativamente mayor $(p<0.00022)$ que la dosis a punto vejiga (ICRU 38) $66.1 \pm 7.6$ Gy (Tablas 1 y 2).

\section{Discusión}

La braquiterapia guiada por imagen en 3D, con el uso de TC y RM, es una práctica clínica recomendada para el tratamiento del cáncer cervicouterino, ya que nos permite realizar la planeación del tratamiento y valorar las dosis entregadas a los tejidos con mayor precisión y exactitud. Las publicaciones actuales sustentan que las imágenes de RM son superiores a las imágenes de TC, debido a la mejor resolución de los tejidos blandos al evaluar la enfermedad residual y la anatomía cervicouterina; sin embargo, al realizar el contorneo de los órganos de riesgo se encontró que los dos estudios de imagen son equivalentes, como lo evidenció el grupo de San Diego ${ }^{12}$. Actualmente GEC-ESTRO recomienda usar imágenes de RM para la delineación del volumen tumoral, sin embargo, debido a que en muchas instituciones no se cuenta con la disponibilidad de este estudio de imagen, se está utilizando la TC para la planificación del tratamiento en $3 \mathrm{D}^{9,10}$.

En este estudio se utilizaron imágenes de TC para realizar el contorneo del CTV-HR y los órganos de riesgo, el volumen tumoral medio hallado $\left(43.03 \pm 12.95 \mathrm{~cm}^{3}\right)$ fue mayor al encontrado en otros estudios en los cuales se realizó el contorneo con $\mathrm{RM}\left(34 \pm 17 \mathrm{~cm}^{3} \text { y } 36 \pm 23 \mathrm{~cm}^{3}\right)^{13,14}$; concluyendo que al contornear con TC se tiende a sobreestimar el CTV-HR. Sin embargo, la cobertura de este aumento de volumen se logró sin exceder las restricciones de dosis para los órganos de riesgo.

Al realizar la planeación en 3D, se comparó la dosis media al $90 \%$ del CTV-HR $(92.1 \pm 2.7 \mathrm{~Gy})$ con la dosis que recibían los puntos A de Mánchester (103.4 $\pm 21.6 \mathrm{~Gy})$ $(p<0.008)$, encontrando que la D90 para el CTV-HR fue menor en la planeación 3D, pero dentro de los límites recomendados por la literatura, ya que basándonos en el reporte de Dimopoulos, et al. informó que una D90 EQD2 = 87 Gy para el CTV-HR está asociada con aproximadamente el 95\% del control local de la enfermedad (Fig. 1) ${ }^{13}$. Mientras que con la planeación a puntos $\mathrm{A}$ de Mánchester las altas dosis de radiación se correlacionan con mayor toxicidad e irradiación a tejidos sanos adyacentes.

Estos resultados se asocian con los volúmenes tumorales mayores a $40 \mathrm{~cm}^{3}$ encontrados en el $61 \%$ de los pacientes de este estudio, en quienes se recomienda el uso de braquiterapia intersticial, lo cual permite obtener una adecuada cobertura de dosis al CTV-HR, sin aumentar la dosis a los tejidos sanos adyacentes ${ }^{13,14}$. Según lo reportado por Derks, et al..$^{15}$, al comparar la braquiterapia 2D vs. la 3D guiada por imágenes, el uso de agujas intersticiales se asoció con un mayor volumen blanco clínico de alto riesgo, una mayor dosis al CTV-HR y una dosis a vejiga más baja. Al comparar las dos técnicas de tratamiento mostró una tendencia con la braquiterapia 3D hacia un mayor control local (93\% a 1 año y $88 \%$ a 3 años) y una mejor supervivencia general (75\% a 3 años y $66 \%$ a 5 años) con toxicidad reducida (17 vs. $12 \%$ ), en comparación con la técnica de braquiterapia $2 \mathrm{D}$ convencional.

El porcentaje de cobertura del volumen tumoral con el $100 \%$ de la dosis prescrita por cada fracción de braquiterapia (5.62 Gy) con la planeación al CTV-HR (97.8 \pm $7.3 \%$ ) fue significativamente mayor $(p<0.000000000043)$ que con la planeación a puntos A de Mánchester (86.6 $\pm 14.7 \%$ ), observándose que la braquiterapia 3D guiada por imágenes proporciona una mejor cobertura tumoral para el CTV-HR comparada con la prescripción a puntos A de Mánchester.

El grupo de $\mathrm{Ha}$, et al.16 encontró una relación entre el tamaño tumoral y la cobertura al CTV-HR, reportando que el valor medio D90 para el CTV-HR fue aproximadamente un $10 \%$ más bajo para los planes basados en $2 \mathrm{D}$ que en los planes en 3D ( 88.4 vs. $97.7 \% ; p=0.068$ ) con tumores $\leq 4 \mathrm{~cm}$, pero cuando tenían tumores $>4 \mathrm{~cm}$ fueron significativamente más altos para los pacientes basados en $3 D$ que para planes basados en $2 \mathrm{D}(96.0 \mathrm{vs.} .78 .1 \% ; p=0.017)$. 


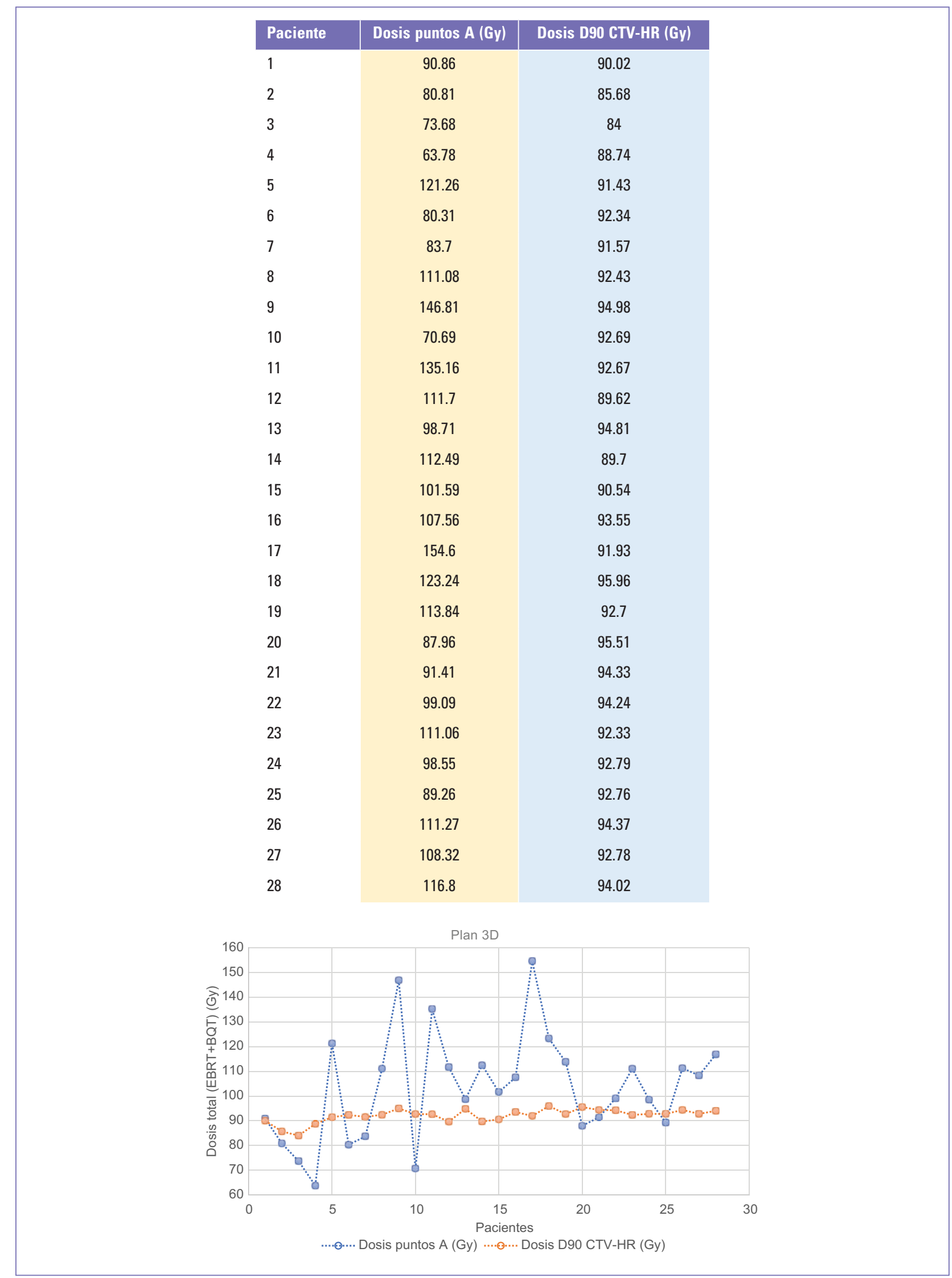

Figura 1. Comparación entre la dosis que reciben los puntos A de Mánchester y el CTV-HR, en la planeación en 3D, por cada paciente. EBRT: external beam radiation therapy (radioterapia de haz externo); CTV-HR: clinical target volume-high risk (volumen blanco clínico de alto riesgo); BOT: braquiterapia; D90: dosis recibida por el $90 \%$ del volumen tumoral. 


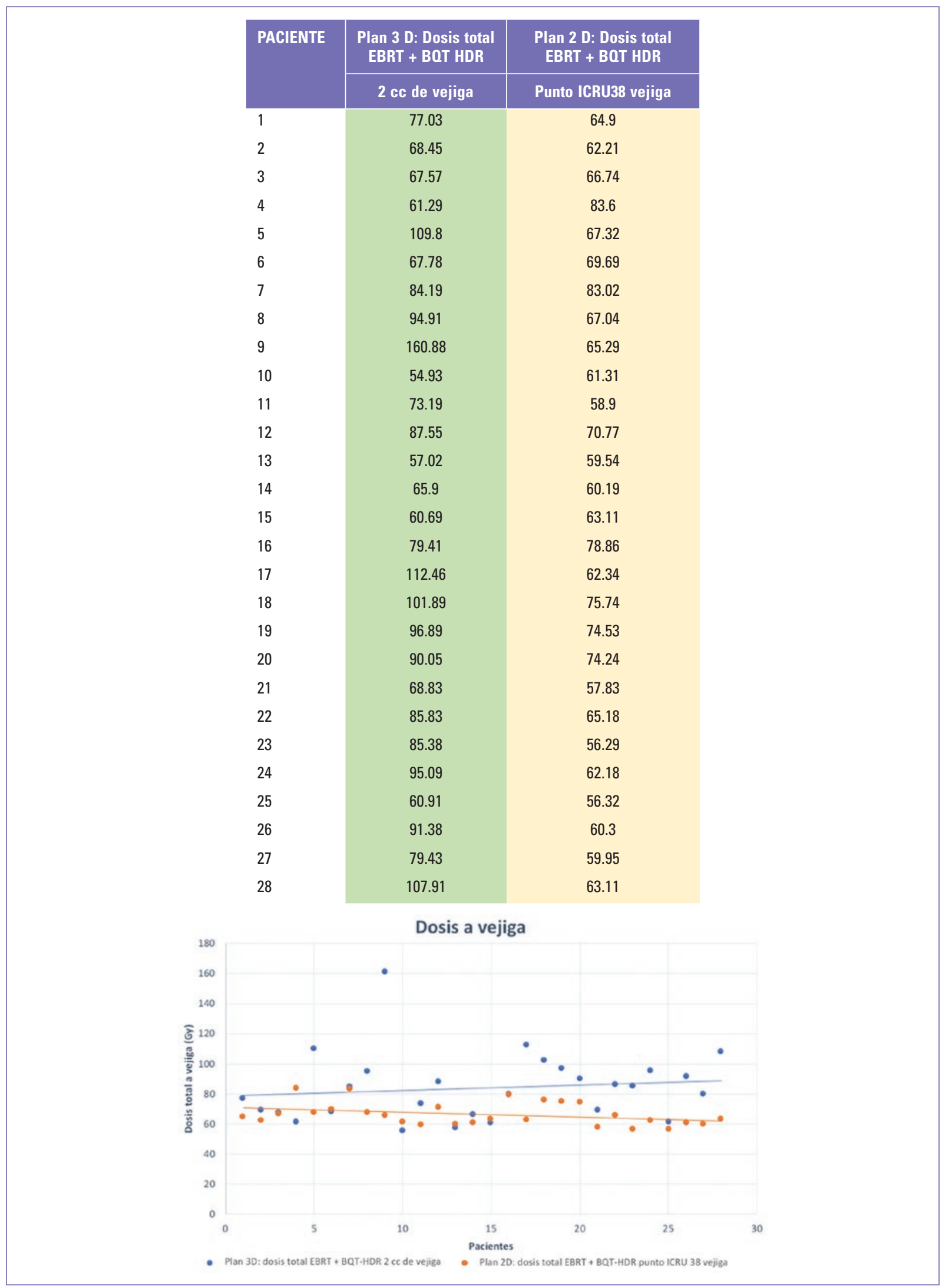

Figura 2. Dosis total (EBRT+BOT) a 2 cc vs. dosis a punto ICRU 38 para la vejiga. EBRT: external beam radiation therapy (radioterapia de haz externo); BOT-HDR: high dose rate brachytherapy (braquiterapia de alta tasa de dosis); ICRU 38: Informe n. ${ }^{0} 38$ de la Comisión Internacional de Unidades de Radiación y Medición. 


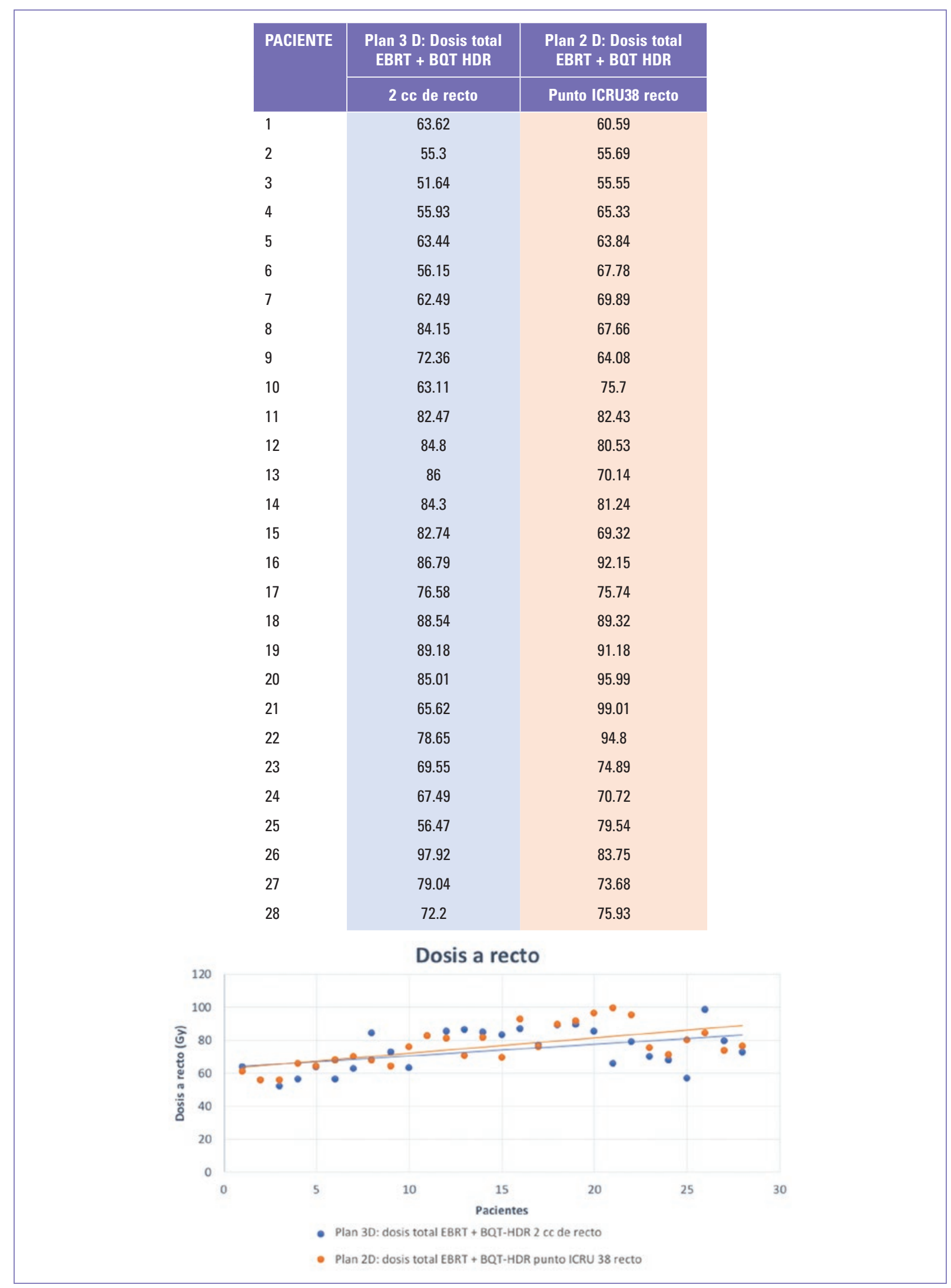

Figura 3. Dosis total (EBRT+BOT) a $2 \mathrm{cc}$ vs. dosis a punto ICRU 38 para el recto. EBRT: external beam radiation therapy (radioterapia de haz externo); BOT: braquiterapia; ICRU 38: Informe n. ${ }^{\circ} 38$ de la Comisión Internacional de Unidades de Radiación y Medición. 
Respecto a los órganos de riesgo los rangos de dosis observados estaban dentro de los límites aceptables y fueron comparables con las dosis informadas por $\mathrm{Kim}^{17}$. La dosis media del punto vejiga ICRU 38 (66.1 $\pm 7.6 \mathrm{~Gy})$ fue notablemente subestimada en comparación con la dosis media a $2 \mathrm{cc}$ de vejiga (83.8 \pm 22.4 Gy) con resultados estadísticamente significativos $(p<0.00577)$ (Fig. 2). Sin embargo, la dosis media del punto recto ICRU $38(75.1 \pm 11.9$ Gy) no difirió significativamente $(p<0.48572)$ de la dosis a $2 \mathrm{cc}$ del recto (73.6 \pm 12.7 Gy) (Fig. 3).

Con los resultados obtenidos se evidencia la viabilidad del enfoque de la planeación basado en TC para la IGBT según las recomendaciones GEC-ESTRO. Sin embargo, debido a la variación en el volumen tumoral contorneado es recomendable, en las instituciones que dispongan de medios suficientes, realizar la simulación con RM, para una mayor exactitud en el contorneo del CTV-HR.

Concluimos que en instituciones en donde se carezca de RM es factible la aplicación de braquiterapia 3D guiada por imagen utilizando TC, ya que la planeación 3D, cuando se compara con la 2D, muestra ser superior en la cobertura tumoral y la delimitación con mayor exactitud de las dosis recibidas en órganos de riesgo.

\section{Financiamiento}

Los autores no recibieron patrocinio para llevar a cabo este artículo.

\section{Conflicto de intereses}

Los autores declaran no tener conflicto de interés alguno.

\section{Responsabilidades éticas}

Protección de personas y animales. Los autores declaran que para esta investigación no se han realizado experimentos en seres humanos ni en animales.

Confidencialidad de los datos. Los autores declaran que en este artículo no aparecen datos de pacientes.

Derecho a la privacidad y consentimiento informado. Los autores declaran que en este artículo no aparecen datos de pacientes.

\section{Bibliografía}

1. Bray F, Ferlay J, Soerjomataran I, Siegel RL, Torre LA, Jemal A. Global Cancer Statistics 2018: GLOBOCAN Estimates of Incidence and Mortality Worldwide for 36 Cancers in 185 Countries. CA Cancer J Clin. 2018:68(6):394-424.

2. Mayadev J, Viswanathan A, Liu Y, Li CS, Albuquerque K, Damato AL, et al. American Brachytherapy Task Group Report: A pooled analysis of clinical outcomes for high-dose-rate brachytherapy for cervical cancer. Brachytherapy. 2017;16(1):22-43.

3. Georg P, Lang S, Dimopoulos JC, Dorr W, Sturdza AE, Berger D, et al. Dose-volume histogram parameters and late side effects in magnetic resonance image-guided adaptive cervical cancer brachytherapy. Int $\mathrm{J}$ Radiat Oncol Biol Phys. 2011;79(2):356-62.

4. Potter R, Georg P, Dimopoulos JC, Grimm M, Berger D, Nesvacil N, et al. Clinical outcome of protocol based image (MRI) guided adaptive brachytherapy combined with 3D conformal radiotherapy with or without chemotherapy in patients with locally advanced cervical cancer. Radiother Oncol. 2011;100(1):116-23.

5. Tod MC, Meredith WJ. A dosage system for use in the treatment of cancer of the uterine cervix. Br J Radio. 1938;11:809.

6. Fletcher $\mathrm{GH}$. Cervical radium applicators with screening in the direction of bladder and rectum. Radiology. 1953;60:77-84.

7. Nag S, Erickson B, Thomadsen B, Orton C, Demanes JD, Petereit D. The American brachytherapy Society recommendations for high-dose-rate brachytherapy for carcinoma of the cervix. Int J Radiat Oncol Biol Phys. 2000;48:201-11.

8. Viswanathan AN, Thomadsen B. American Brachytherapy Society Cervical Cancer recommendations Committee; American Brachytherapy Society consensus guidelines for locally advanced carcinoma of the cervix. Part I: General principles. Brachytherapy. 2012;11(1):33-46.

9. Haie-Meder C, Potter R, van Limbergen E, Briot E, de Brabandere M, Dimopoulos J, et al. Recommendations from Gynaecological (GYN) GEC-ESTRO Working Group (I): concepts and terms in 3D image based $3 D$ treatment planning in cervix cancer brachytherapy with emphasis on MRI assessment of GTV and CTV. Radiother Oncol. 2005;74(3):235-45.

10. Potter R, Haie-Meder C, van Limbergen E, Barillot I, de Brabandere M, Dimopoulos J, et al. Recommendations from Gynaecological (GYN) GEC ESTRO Working Group (II): concepts and terms in 3D image-based treatment planning in cervix cancer brachytherapy-3D dose volume parameters and aspects of $3 \mathrm{D}$ image-based anatomy, radiation physics, radiobiology. Radiother Oncol. 2006;78:67-77.

11. Wyckoff HO, Allisy A, Caswell RS, Adams ED, Cowper G, Edholm P. International Commission on Radiation Units and Measurements. ICRU REPORT 38. Dose and Volume Specification for reporting Intracavitary Therapy in Gynecology. Bethesda, Maryland, EE.UU.: International Commission on Radiation Units \& Measurements; 1985.

12. Eskander RN, Scanderbeg D, Saenz CC, Brown M, Yashar C. Comparison of computed tomography and magnetic resonance imaging in cervical cancer brachytherapy target and normal tissue contouring. Int $\mathrm{J}$ Gynecol Cancer. 2010;20:47Y53.

13. Dimopoulos J, Lang S, Kirisits C, Fidarova E, Berger D, Georg P, et al. Dose volume histogram parameters and local tumor control in magnetic resonance image-guided cervical cancer brachytherapy. Int J Radiat Oncol Biol Phys. 2009;75:56-63.

14. Kirisits C, Pötter R, Lang S, Dimopoulos J, Wachter-Gerstner N, Georg D. Dose and volume parameters for MRI based treatment planning in intracavitary brachytherapy of cervix cancer. Int J Radiat Oncol Biol Phys. 2005;62:901-11.

15. Derks K, Steenhuijsen JLG, van den Berg HA, Houterman S, Cnossen J, Haaren PV, et al. Impact of brachytherapy technique (2D vs. 3D) on outcome following radiotherapy of cervical cancer. J Contemp Brachytherapy. 2018;10(1):17-25.

16. Ha IB, Jeong BK, Kang KM, Jeon $\mathrm{H}$, Lee $\mathrm{YH}$, Choi HS, et al. Who really benefits from $3 \mathrm{D}$-based planning of brachytherapy for cervical cancer? J Korean Med Sci. 2018;33(18):e135

17. Kim RY, Shen S, Duan J. Image-based three-dimensional treatment planning of intracavitary brachytherapy for cancer of the cervix: Dose-volume histograms of the bladder, rectum, sigmoid colon, and small bowel. Brachytherapy. 2007;6(3):187-94. 